\title{
Nanoscale
}

D) Check for updates

Cite this: Nanoscale, 2020, 12 18600

Received 31st May 2020,

Accepted 14th August 2020

DOI: $10.1039 /$ dOnr04179c

rsc.li/nanoscale

\section{Elucidating the mechanism of the surface functionalization dependent neurotoxicity of graphene family nanomaterials $\uparrow$}

\author{
Zhiling Guo, (iD) a,b,c Peng Zhang, (D) *c Andrew J. Chetwynd, (iD) ${ }^{c}$ \\ Heidi Qunhui Xie, ${ }^{a, b, d}$ Eugenia Valsami-Jones, (D) ${ }^{c}$ Bin Zhao*a,b,d and \\ Iseult Lynch iD c
}

Graphene family nanomaterials (GFNs) have shown great potential for biological and environmental applications; however, their future use has been debated due to their reported potential neurotoxicity. Moreover, the effects of surface functionalization on their biological end points are largely unknown. Here, we compared the effects of reduced graphene oxide (RGO), and carboxylated (G-COOH), hydroxylated (G-OH) and aminated $\left(\mathrm{G}-\mathrm{NH}_{2}\right)$ graphene nanosheets on human neuroblastoma cells (SK-N-SH). All GFNs inhibited cellular growth at concentrations of $0.1-10 \mathrm{mg} \mathrm{L}^{-1}$ after $24 \mathrm{~h}$ exposure. The toxicity was attenuated over longer exposure times, with the exception of $\mathrm{G}-\mathrm{NH}_{2}$. Although the overall acute toxicity followed the order: G-OH $\approx \mathrm{G}-\mathrm{COOH}>\mathrm{RGO}>\mathrm{G}-\mathrm{NH}_{2}, \mathrm{G}-\mathrm{NH}_{2}$ induced more persistent toxicity and more metabolic disturbance compared to the other GFNs, with lipid and carbohydrate metabolism being the most affected. The potential for physical disruption of the lipid membrane and oxidative damage induced by GFNs varied with different functionalization, which accounts for the observed differences in neurotoxicity. This study provides significant insights into the neurological effects of GFNs, and suggests that $\mathrm{G}-\mathrm{NH}_{2}$ is not as safe as reported in many previous studies. The neurological effect of GFNs over longer term exposure should be considered in future studies.

\section{Introduction}

Graphene based nanomaterials (GFNs) have attracted great research interest in recent years due to their unique optical,

\footnotetext{
${ }^{a}$ State Key Laboratory of Environmental Chemistry and Ecotoxicology,

Research Center for Eco-Environmental Sciences, Chinese Academy of Sciences, Beijing 100085, China. E-mail: binzhao@rcees.ac.cn

${ }^{b}$ University of Chinese Academy of Sciences, Beijing 100049, China

${ }^{c}$ School of Geography, Earth and Environmental Sciences, University of Birmingham, Birmingham B15 2TT, UK.E-mail: p.zhang.1@bham.ac.uk

${ }^{d}$ Institute of Environment and Health, Hangzhou Institute for Advanced Study, University of Chinese Academy of Sciences, Hangzhou 310024, China

$\dagger$ Electronic supplementary information (ESI) available: Experimental details, Raman spectra, size, height, hydrodynamic size and zeta potential of the GFNs, MDA content, differentially regulated metabolites, and metabolite set enrichment analysis. See DOI: 10.1039/d0nr04179c
}

electronic, mechanical and thermal properties. ${ }^{1}$ GFNs have great potential in the biomedical sector for drug delivery, bioimaging, phototherapy etc. ${ }^{2}$ As a 2-D material, graphene has been reported as an ideal substrate to support cell attachment, proliferation and differentiation. ${ }^{3}$ Recent studies have shown that GFNs might also be used as a platform to enhance cellular growth such as that of neuronal cells. ${ }^{4}$ However, the potential neurotoxicity of GFNs has also been reported in different biological models such as human neuroblastoma cells (SH-SY5Y), ${ }^{5}$ Caenorhabditis elegans,${ }^{6}$ or zebrafish. $^{7}$ For example, graphene oxide (100-800 nm) at concentrations higher than $80 \mathrm{mg} \mathrm{L}^{-1}$ can cause neurotoxicity to SH-SY5Y cells. ${ }^{5}$ Graphene oxide at environmentally relevant concentrations $\left(0.01-1 \mu \mathrm{g} \mathrm{L}^{-1}\right)$ can induce Parkinson's disease-like symptoms in zebrafish larvae. ${ }^{7}$ Clearly, more studies are required to fully understand the neurological effects of GFNs before they can be used safely for clinical and tissue engineering applications.

GFN-induced cytotoxicity mainly involves physical damage and oxidative stress. The sharp edge of GFNs may penetrate the cell membrane and extract membrane lipids, thereby causing membrane damage. ${ }^{8}$ GFNs may also generate free radicals ${ }^{9}$ or oxidize antioxidants ${ }^{10}$ in cells, causing oxidative stress. The biological effects of GFNs and the mechanisms of action are highly dependent upon their physicochemical properties such as lateral size, shape, thickness, surface charge, and surface functionalization. ${ }^{11}$ Graphene oxide with larger lateral sizes can wrap around the cell surface in nutrient rich medium, thereby preventing cells from acquiring essential nutrients from the culture medium. ${ }^{12}$ Positively charged GFNs might bind more easily to the negatively charged phosphate headgroups in the phospholipid membrane, causing cell membrane damage. ${ }^{13}$

The aim of this study is to understand how surface functionalization affects the biological outcomes of GFNs on neuronal cells. Specifically, we compared the effects of reduced graphene oxide (RGO), and carboxylated (G-COOH), hydroxylated (G-OH) and aminated $\left(\mathrm{G}-\mathrm{NH}_{2}\right)$ graphene 
nanosheets on the growth of human neuroblastoma cells (SK-N-SH). The potential of GFNs to induce physical disruption of the cell membranes and cellular oxidative damage was evaluated to understand the difference in the mechanisms of action between different functionalisations. Moreover, the metabolic disturbance to the cells induced by the four GFNs was evaluated using a metabolomics approach. All four GFNs showed neurotoxicity to the SK-N-SH cells although to different extents. Both physical disruption and oxidative stress were involved in the toxicity. G- $\mathrm{NH}_{2}$ showed less acute but more persistent toxicity and induced more metabolic disturbance of the cells than the other GFNs.

\section{Results and discussion}

RGO, G-OH, G-COOH and $\mathrm{G}-\mathrm{NH}_{2}$ all have similar lateral sizes which were $0.14 \pm 0.04 \mu \mathrm{m}, 0.16 \pm 0.06 \mu \mathrm{m}, 0.14 \pm 0.04 \mu \mathrm{m}$ and $0.15 \pm 0.03 \mu \mathrm{m}$, respectively (Fig. 1A; Table S1†). The thicknesses of RGO $(0.91 \pm 0.15 \mathrm{~nm}), \mathrm{G}-\mathrm{OH}(0.90 \pm 0.16 \mathrm{~nm})$, G-COOH $(0.89 \pm 0.23 \mathrm{~nm})$, and $\mathrm{G}-\mathrm{NH}_{2}(0.95 \pm 0.14)$ obtained from the AFM images were also similar (Fig. 1A), suggesting that all the four GFNs were triple layered. The defects and disorder in the materials, indicated by $I_{\mathrm{d}} / I_{\mathrm{g}}$ ratios (high ratio indicates high disorder and defect ${ }^{14}$ ) in the Raman spectra followed the order: $\mathrm{G}-\mathrm{NH}_{2}>\mathrm{G}-\mathrm{COOH}>\mathrm{G}-\mathrm{OH}>\mathrm{RGO}$ (Fig. $\mathrm{S} 1 \dagger$ ). The hydrodynamic sizes of the four GFNs in deionized water showed no significant difference (Table S1†), suggesting that their agglomeration states were similar. The RGO, G-OH and G-COOH were negatively charged, while $\mathrm{G}-\mathrm{NH}_{2}$ was positively charged in deionized water (Table $\mathrm{S} 1 \dagger$ ), due to the presence of different functional groups. XPS analysis showed that the $\mathrm{RGO}, \mathrm{G}-\mathrm{OH}$ and $\mathrm{G}-\mathrm{COOH}$ were composed of $\mathrm{C}$ and $\mathrm{O}$, and G-NH $\mathrm{N}_{2}$ was composed of $\mathrm{C}, \mathrm{O}$ and $\mathrm{N}$ (Fig. 1B). The atomic percent of $\mathrm{C}, \mathrm{N}$ and $\mathrm{O}$ in the materials is shown in Table S1. $\dagger$ Since the primary lateral size, thickness and hydrodynamic size of the four types of graphene were all similar, any difference in the cellular effects between the GFNs can be attributed to the difference in the functional groups.
We initially compared the neurotoxic potential of the GFNs over 72 h, with cell viability tested at 24, 48 and 72 h (Fig. 2A). All materials showed inhibitory effects on the cellular growth of SK-N-SH cells at a concentration of $0.1 \mathrm{mg} \mathrm{L} \mathrm{L}^{-1} \cdot \mathrm{G}^{-\mathrm{NH}_{2}}$ showed the lowest toxic response with $76 \%$ cell survival at $24 \mathrm{~h}$, while only $54-59 \%$ of cells survived in the RGO, G-OH, and $\mathrm{G}-\mathrm{COOH}$ groups. Interestingly, with increased exposure time, the cell viability in the $\mathrm{RGO}, \mathrm{G}-\mathrm{OH}$, and $\mathrm{G}-\mathrm{COOH}$ groups increased, while for the $\mathrm{G}-\mathrm{NH}_{2}$ group it remained stable (76-80\%). After $72 \mathrm{~h}$ exposure, the cell viability in the RGO and G-COOH groups increased to $\sim 100 \%$, while the cell viability in the $\mathrm{G}-\mathrm{OH}$ group increased to $77.6 \%$. The cell viability did not show a significant change when the exposure concentration was increased to $1 \mathrm{mg} \mathrm{L}^{-1}$ in the $\mathrm{RGO}, \mathrm{G}-\mathrm{OH}$, and G- $\mathrm{NH}_{2}$ groups, while the cell viabilities in the $\mathrm{G}-\mathrm{COOH}$ group decreased by $13 \%$ and $8 \%$ at 48 and $72 \mathrm{~h}$, respectively, com-

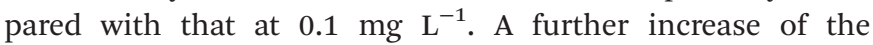
exposure concentration to $10 \mathrm{mg} \mathrm{L^{-1 }}$ caused a significant decrease of the cell viability, with $42 \%, 28 \%$ and $23 \%$ survival rate at $24 \mathrm{~h}$ for $\mathrm{RGO}, \mathrm{G}-\mathrm{OH}$, and $\mathrm{G}-\mathrm{COOH}$, respectively, and increased to $66 \%, 43 \%$ and $49 \%$, respectively, after $72 \mathrm{~h}$ exposure. However, increasing the $\mathrm{G}^{-\mathrm{NH}_{2}}$ exposure concentration did not affect the cell viability compared to the initial $0.1 \mathrm{mg} \mathrm{L}^{-1}$ exposure.

In summary, the neurotoxic potential of the four GFNs followed the order: $\mathrm{G}-\mathrm{OH} \approx \mathrm{G}-\mathrm{COOH}>\mathrm{RGO}>\mathrm{G}-\mathrm{NH}_{2}$. The lower toxicity observed for $\mathrm{G}-\mathrm{NH}_{2}$ is consistent with previous studies which showed that positively charged aminated graphene was more favorable for neuronal growth. ${ }^{15}$ It was proposed that amine modification can reduce the charge transfer to cells, thus reducing the toxicity of graphene. ${ }^{16}$ However, in our study, G- $\mathrm{NH}_{2}$ showed sustained neurotoxicity over the exposure period, which was distinct from the adaptive response of the SK-N-SH cells observed for other three GFN groups, suggesting that $\mathrm{G}-\mathrm{NH}_{2}$ may cause persistent neurotoxicity.

To further elucidate the different patterns of the neurotoxicity induced by the GFNs, we examined whether exposure to the GFNs induces membrane damage and/or oxidative stress in the cells. All of the GFNs induced membrane damage as
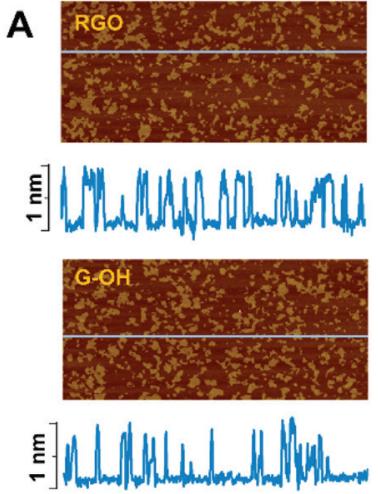
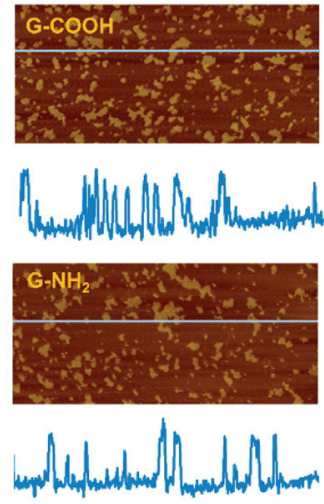

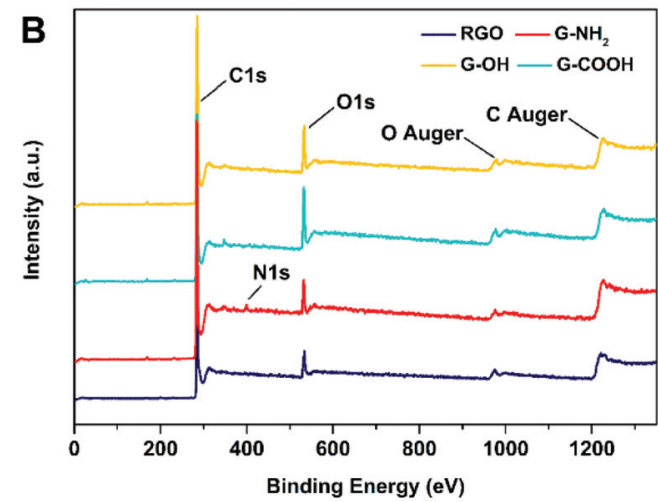

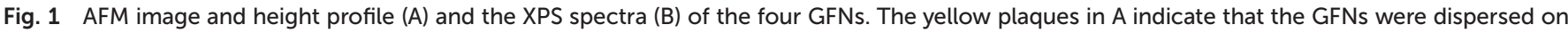
silicon wafers. The blue horizontal lines (with a length of $2 \mu \mathrm{m}$ ) in the AFM images indicate the regions analyzed for the height profiles. 

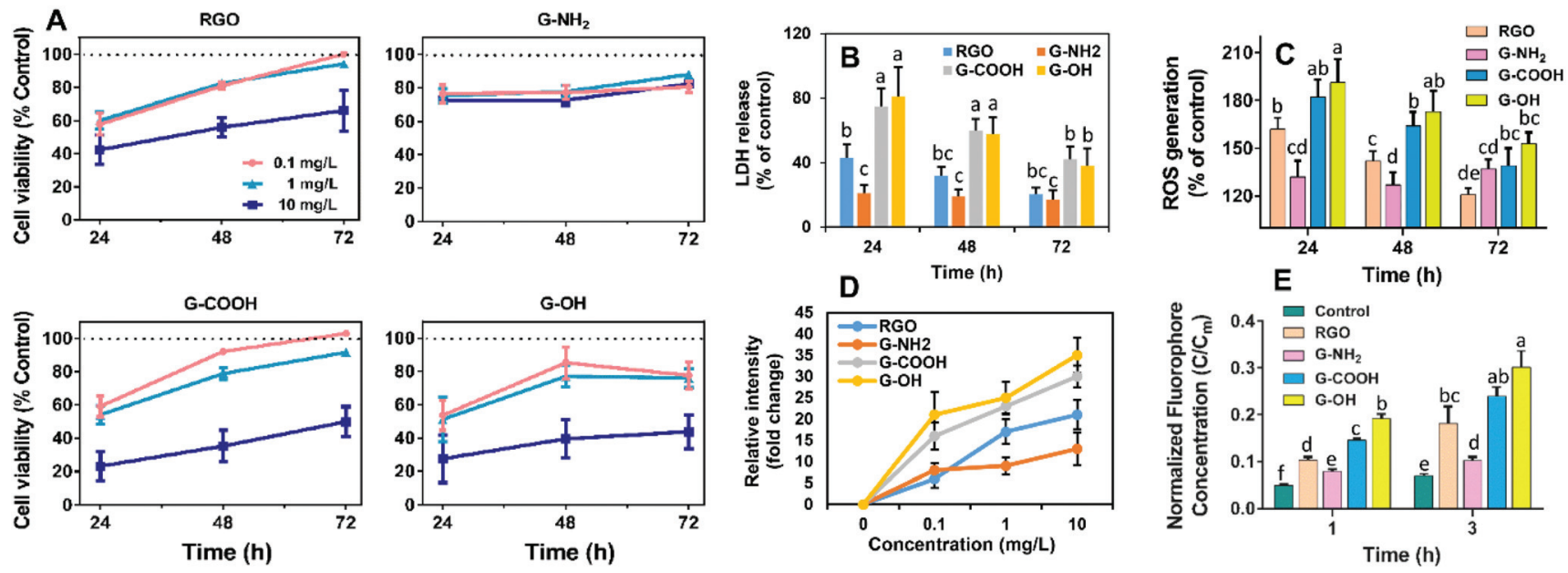

Fig. 2 (A) Cell viability after treatment with four GFNs for 24, 48 and $72 \mathrm{~h}$. (B) LDH release from SK-N-SH cells. (C) ROS accumulation (\% of control) in SK-N-SH cells. (D) Oxidative potential of the GFNs by measuring the time-dependent oxidation of DCFH. (E) Potential of graphene-induced physical disruption of lipid membranes by measuring the fluorophore leakage from the liposome vesicles. All data are expressed as mean \pm SD $(n=6)$. Different lowercase letters indicate significant difference at $p<0.05$.

demonstrated by the leakage of LDH (Fig. 2B) and lipid peroxidation (Fig. S2†). The extent of the damage followed the same trend as the cell viability assay: $\mathrm{G}-\mathrm{OH} \approx \mathrm{G}-\mathrm{COOH}>\mathrm{RGO}>$ G-NH $\mathrm{N}_{2}$, suggesting that the graphenes caused different degrees of membrane damage. Adaptive responses were also observed, with the membrane damage being alleviated after $72 \mathrm{~h}$ of exposure to G-OH, G-COOH and RGO; however, $\mathrm{G}^{-\mathrm{NH}_{2}}$ caused sustained LDH release throughout the $72 \mathrm{~h}$ exposure period.

The membrane damage may be due to a reaction between membrane lipids and excess free radicals or be the result of physical disruption by the sharp edge of the graphene sheets. Indeed, overaccumulation of ROS in the cells was observed in all the treatments in the same patterns as the cell viability (Fig. 2C). GFNs may also cause oxidative damage due to their intrinsic oxidative potential. ${ }^{17,18}$ Fig. 2D shows that the four GFNs exhibited intrinsic oxidative potential, evidenced by their ability to oxidize DCFH. G-NH $\mathrm{N}_{2}$ demonstrated a higher oxidative potential than the other materials, which is consistent with its higher disorder and the defects observed by Raman spectroscopy (Fig. S1†). However, this is inconsistent with the results of the cellular toxicity of $\mathrm{G}-\mathrm{NH}_{2}$ being lower than the other GFNs, suggesting that the mechanism of neurotoxicity is not solely explained by the direct oxidation of cells by GFNs. Previous studies reported that graphene may cause membrane damage by direct physical insertion. ${ }^{19}$ We therefore examined this possibility and found that the GFNs have different capacities for causing membrane damage to liposome vesicles. The extent of leakage of the fluorophore from vesicles followed the same trend as that of cell viability and membrane damage (Fig. 2E). These results suggest that physical disruption of the membrane lipids, rather than the direct oxidation by the GFNs, is the main mechanism of neurotoxicity, and that surface functionalization affected their capacity for disrupting lipid membranes and the intrinsic oxidative potentials of GFNs.
To obtain additional molecular mechanisms for the differential patterns of neurotoxicity triggered by the GFNs, non-targeted metabolomics was carried out. The score plot of the partial least-squares discriminant analysis (PLS-DA) showed a clear separation between the control and treatment groups, indicating that all treatments caused perturbation in the metabolic profiles of SK-N-SH cells. Moreover, all treatment groups could be discriminated from each other except for $\mathrm{G}-\mathrm{COOH}$ versus G-OH (Fig. 3A), which correlates with the cytotoxicity data indicating that $\mathrm{G}-\mathrm{COOH}$ and $\mathrm{G}-\mathrm{OH}$ induced similar inhibitory effects on neuronal growth. The discriminating metabolites that lead to the separation of the control and treatment groups (VIP $>1$ and $p<0.05$ ) were isolated and identified (Fig. S3†). More metabolites were altered by RGO and G-NH than G-COOH and G-OH (Fig. S3†). In total, 35, 31, 8 and 14 metabolites were significantly altered by $\mathrm{RGO}, \mathrm{G}-\mathrm{NH}_{2}$, G-COOH, and G-OH, respectively. Following exposure to RGO, G-COOH, and G-OH, all the altered metabolites were downregulated, while for the G-NH $\mathrm{N}_{2}$ exposed cells, 19 metabolites were upregulated and 12 were downregulated (Fig. S3 $\dagger$ ). Only a few metabolites (1-6) overlapped among the treatments (Fig. 3B). Treatment-specific metabolites accounted for $65.7 \%$ and $67.7 \%$ in $\mathrm{RGO}$ and $\mathrm{G}-\mathrm{NH}_{2}$ groups, respectively (Fig. 3B). The perturbed metabolites were predominantly lipids, carbohydrates, nucleotides, and amino acids (Fig. 3C). Functional enrichment analysis showed that the disturbed metabolites in each treatment participate in carbohydrate metabolism, lipid biosynthesis, and antioxidant metabolism (Fig. S4 $\dagger$ ). The four GFNs induced different patterns of alterations in metabolites that are related to those pathways (Fig. 3D). In particular, metabolites related to energy production were differentially altered by the GFNs. Glycolytic intermediates such as glucose, glycerol 3-phosphate, and UDP- $N$-acetylglucosamine were significantly decreased in the RGO group, while they were not affected by the other GFNs. Metabolites that participate in oxi- 
A

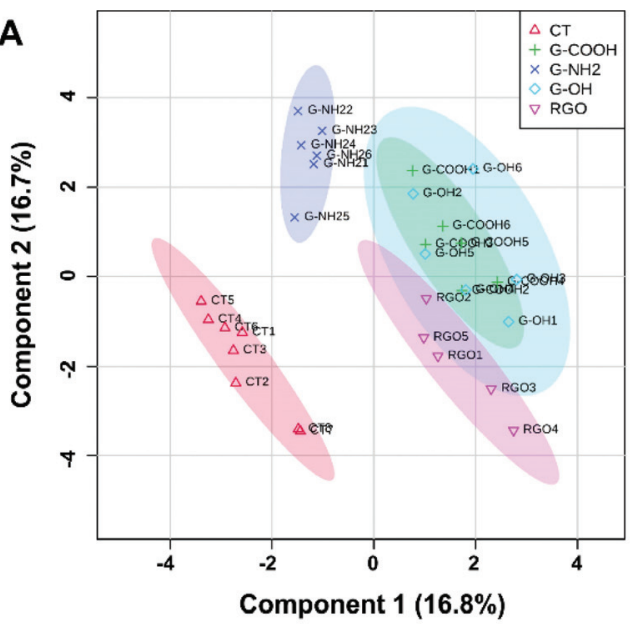

B

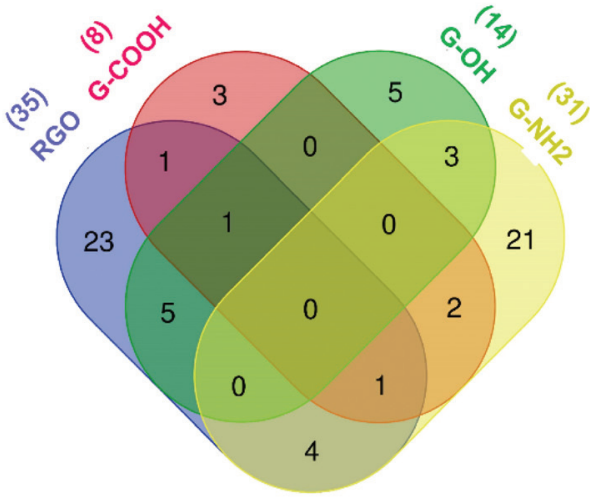

C

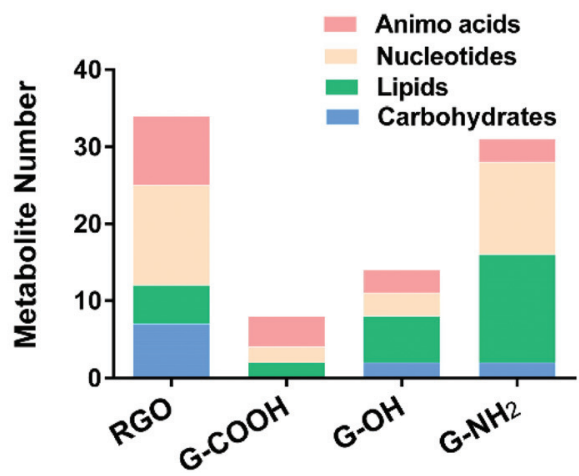

D
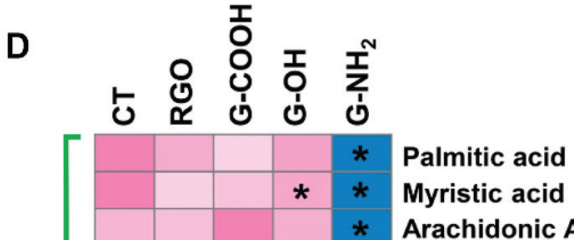

Arachidonic Acid

Dodecanoic Acid

Pentadecanoic Acid

Oleic Acid

Linoleic Acid

LysoPC(18:1(9Z))

MG(16:0)

PG(16:0/18:1(9Z))

Cis- $(6,9,12)$-Linolenic acid

L-Palmitoylcarnitine

Glycerol 3-phosphate

Choline

sn-Glycerol 3-phosphoethanolamine

Glycerophosphocholine

Guanosine 5'-triphosphate (GTP)

Adenosine 5'-triphosphate (ATP)

Adenosine 5'-diphosphate (ADP)

Nicotinamide

1-Methylnicotinamide

Flavin mononucleotide (FMN)

ADP-ribose

Flavin adenine dinucleotide (FAD)

UDP-N-acetylglucosamine

UDP-D-Galactose

D-Mannose-6-phosphate

D-Mannose 1-phosphate

N-Acetylglucosamine 1-phosphate

D-Mannose

Alpha-D-Glucose

L-Gulonic gamma-lactone

Taurine

\section{$\operatorname{Max}$}

Fig. 3 PLS-DA score plot of metabolic profiles in SK-N-SH cells after $72 \mathrm{~h}$ treatment with $10 \mathrm{mg} \mathrm{L}^{-1}$ of the four GFNs (A). Venn diagram analyzed using an online tool (http://bioinformatics.psb.ugent.be/webtools/Venn/) shows the overlap of perturbed metabolites in the different treatments (B). The number of perturbed amino acids, nucleotides, lipids and carbohydrates in each treatment (C). Relative levels of metabolites involved in lipid biosynthesis, carbohydrate metabolism, and antioxidant metabolism after GFN exposure. Rows indicate metabolites. The degree of change in the metabolite concentration following GFN exposure is color-coded. * indicates significant difference when compared with the control (CT).

dative phosphorylation were downregulated in the RGO (e.g. ADP, nicotinamide, 1-methylnicotinamide) and G-COOH (e.g. 1-methylnicotinamide) groups but were upregulated in the G-NH $\mathrm{NH}_{2}$ group (e.g. ATP, FMN, FAD) (Fig. 3D). Additionally, all treatments induced decreased lipid biosynthesis although the discriminating lipids were different. Moreover, the antioxidant activity was differently affected. For example, L-gulonic gamma lactone, an essential substrate for the biosynthesis of the antioxidant ascorbic acid, ${ }^{20}$ was decreased by RGO treatment. Taurine, a critical component involved in antioxidative defense, ${ }^{21}$ was decreased in the $\mathrm{G}-\mathrm{COOH}$ group but increased in the $\mathrm{G}-\mathrm{NH}_{2}$ group. These results indicate that different surface treatments of otherwise identical GFNs exert different effects on the metabolism of SK-N-SH cells. 
Previous studies suggest that GFNs may trigger the mitochondrial pathway and activate caspase-3 and PARP, which eventually lead to cell apoptosis. ${ }^{22}$ The mitochondria generated superoxide can result in the loss of mitochondrial membrane potential, which impairs the electron transfer chain and energy metabolism. It was reported that few-layer graphene can increase the cytoplasm $\mathrm{Ca}^{2+}$ concentration and thus cause depolarization of the mitochondrial membrane and increase the membrane permeability and eventually initiate apoptosis. $^{23}$ Additional signaling pathways including MAPKs and TGF- $\beta$ might be also triggered by GFNs. ${ }^{22}$ GFNs may trigger JNK which activates the $\mathrm{Bc1}-2$ protein family proapoptotic members (Bim and Bax) followed by the activation of MOMP and caspase- 3 and subsequent cell apoptosis. ${ }^{24}$ These signaling pathways may also be involved in the neurotoxicity of the GFNs observed in this study, which need further studies in the future.

\section{Conclusion}

In summary, this study found that surface functionalization affects the neurotoxic potential of graphene. All GFNs caused neurotoxicity even at the lowest concentration $\left(0.1 \mathrm{mg} \mathrm{L} \mathrm{L}^{-1}\right)$. The toxicity is greater than that previously reported by $\mathrm{Lv}$ et al. where the authors found the neurotoxicity of GO to SH-SY5Y cells (a subclone of the SK-N-SH cells) at a concentration higher than $80 \mathrm{mg} \mathrm{L^{-1 } . 5}$ This might be due to the different sizes and surface oxygen contents of the GFNs in the two studies, as well as different sensitivities of the cell lines that were used. It is interesting that G-COOH and $\mathrm{G}-\mathrm{OH}$ caused more severe acute toxicity than RGO and G-NH$H_{2}$, while $\mathrm{RGO}$ and $\mathrm{G}-\mathrm{NH}_{2}$ caused more disturbance in the cellular metabolism. Moreover, the toxicity of G-COOH, G-OH and RGO was all alleviated over longer exposure times; however, the toxicity of $\mathrm{G}-\mathrm{NH}_{2}$ remained constant, suggesting that $\mathrm{G}-\mathrm{NH}_{2}$ may cause longer chronic neurotoxicity than the other materials. Our results indicate that caution should be taken in developing and testing GFNs as a general enhancer for cellular growth. Physicochemical properties such as the particle size, surface functionalization and sensitivity of the specific cell lines should all be considered during the study for safe-by-design of GFNs for environmental and biological application. Moreover, longer term studies are required to evaluate the chronic effects of GFNs on cell growth.

\section{Methods}

\subsection{Materials and characterization}

RGO, G-COOH, G-OH and $\mathrm{G}-\mathrm{NH}_{2}$ nanosheets were procured from Chengdu Organic Chemicals Co. Ltd (Chengdu, China). The morphology, lateral size and thickness of the GFNs were measured by atomic force microscopy (AFM) (Veeco Dimension 3100, USA). The chemical structures were deter- mined by XPS (ESCALAB 250Xi, Thermo Scientific, USA) equipped with a twin-crystal monochromatic X-ray source. The hydrodynamic sizes of the GFNs $\left(10 \mathrm{mg} \mathrm{L}^{-1}\right.$, suspension in deionized water) were measured using Dynamic Light Scattering (DLS) and the $\zeta$ potentials of the GFN suspensions were measured using a ZetaSizer Nano ZS system (Malvern Instruments, UK).

\subsection{Cell culture}

SK-N-SH cells were purchased from the cell resource center of the Chinese Academy of Medical Sciences (Beijing, China). Cells (passage 3-passage 5 after defrosting) were maintained in Dulbecco's modified Eagle's medium (DMEM), supplemented with $10 \%$ fetal bovine serum (FBS), $100 \mathrm{IU} \mathrm{mL}^{-1}$ penicillin and $100 \mu \mathrm{g} \mathrm{mL} \mathrm{m}^{-1}$ streptomycin, and incubated at $37{ }^{\circ} \mathrm{C}$ in a water-saturated $5 \% \mathrm{CO}_{2}$ incubator. All reagents for cell culture were obtained from Invitrogen (Carlsbad, CA, USA).

\subsection{Cell counting kit-8 (CCK-8) assay}

For cell viability tests, cultured SK-N-SH cells in 96-well plates $\left(1 \times 10^{4}\right.$ cells per well) were treated with the four GFNs at concentrations of $0,0.1,1$, and $10 \mathrm{mg} \mathrm{L}^{-1}$ for 24,48 and $72 \mathrm{~h}$. Cell viability was measured using the CCK-8 kit (Abcam, UK) according to the manufacturer's instructions.

\subsection{ROS content}

Total reactive oxygen species (ROS) contents in the cells were measured by the DCFH-DA $\left(2^{\prime}, 7^{\prime}\right.$-dichlorodihydrofluorescein diacetate) staining method according to the manufacturer's instruction (Nanjing Jiancheng Ltd, China).

\subsection{Dye-leakage experiment}

Physical damage of the cell membrane by the GFNs was evaluated via a dye-leakage experiment following the method described previously. ${ }^{25}$ Briefly, monodisperse DOPC (1,2-dioleoyl-sn-glycero-3-phosphocholine) liposomes of $100 \mathrm{~nm}$ encapsulating carboxyfluorescein dye were prepared using extrusion methods (Avanti Polar Lipids, Inc). The liposomes were then exposed to $10 \mathrm{mg} \mathrm{L}^{-1}$ of the different GFNs. The physical disruption of the liposome membrane was evaluated by measuring the concentration of the carboxyfluorescein leaking out from the liposomes. Details of the experiments are presented in the ESI. $\dagger$

\subsection{Intrinsic oxidative potential}

The intrinsic oxidative potential of the GFNs was evaluated by a method described previously by Zhang et al. ${ }^{18}$ Briefly, $2^{\prime}, 7^{\prime}-$ dichlorofluorescin diacetate (DCFH-DA) was hydrolyzed in $1 \mathrm{M}$ $\mathrm{NaOH}$ for $30 \mathrm{~min}$ in the dark. The solution was then neutralized with phosphate buffer solution (PBS, pH 7.4). The obtained DCFH $(30 \mu \mathrm{M})$ was then mixed with GFN suspensions $\left(0.1,1,10 \mathrm{mg} \mathrm{L}^{-1}\right)$ for $24 \mathrm{~h}$. The fluorescence spectra and intensity were then measured at $500-600 \mathrm{~nm}$ on a microplate reader (TECAN Infinite 200 Pro, Switzerland) at an excitation wavelength of $490 \mathrm{~nm}$. 


\subsection{Metabolite profiling}

The cells were seeded in a 6-well-plate at 500000 cells per well $24 \mathrm{~h}$ before exposure to GFNs. Four different GFNs were employed individually at a concentration of $10 \mathrm{mg} \mathrm{L}^{-1}$. Seventy-two hours after treatment, the cells were washed three times with phosphate buffered saline $(1 \times$ PBS $)$, followed by addition of $1 \mathrm{~mL}$ of methanol/acetonitrile/ $\mathrm{H}_{2} \mathrm{O}(2: 2: 1, \mathrm{v} / \mathrm{v} / \mathrm{v})$. Samples were ultrasonicated for $30 \mathrm{~min}$ at $4{ }^{\circ} \mathrm{C}$ twice, and then kept at $-20{ }^{\circ} \mathrm{C}$ for $1 \mathrm{~h}$ for protein precipitation. The precipitates were removed by centrifugation at $13000 \mathrm{rpm}$ at $4^{\circ} \mathrm{C}$ for $15 \mathrm{~min}$. The supernatants were collected, lyophilized, and stored at $-80{ }^{\circ} \mathrm{C}$ prior to metabolomics analysis. QC samples were prepared by mixing equal volumes of each sample. Untargeted metabolomic analysis was carried out using ultraperformance liquid chromatography quadrupole time-of-flight tandem mass spectrometry (UHPLC-QTOF-MS/MS). Details of the instrumentation and data analysis are described in the ESI. $\dagger$

\section{Author contributions}

Z. G., P. Z. and B. Z. designed the study. Z. G. and P. Z. performed the experiments and wrote the manuscript. I. L., A. C., H. Q. X. and E. V. J. revised the manuscript. B. Z. provided the funding support.

\section{Conflicts of interest}

The authors declare no competing financial interests.

\section{Acknowledgements}

This work was supported by the National Natural Science Foundation of China (Grant No. 21836004, 91543204, and 21525730), the Strategic Priority Research Program of the Chinese Academy of Sciences (Grant No. XDB14030400), and the National Key Research and Development Program of China (Grant No. 2018YFA0901103). The Marie Skłodowska-Curie Individual Fellowship (NanoBBB Grant Agreement No. 798505 to Z. Guo) under the European Union's Horizon 2020 research program was acknowledged. Technical support from the Shanghai Applied Protein Technology facility (Shanghai, China) is gratefully acknowledged.

\section{References}

1 C. Soldano, A. Mahmood and E. Dujardin, Carbon, 2010, 48, 2127-2150.

2 S. Song, H. Shen, Y. Wang, X. Chu, J. Xie, N. Zhou and J. Shen, Colloids Surf., B, 2020, 185, 110596.
3 O. N. Ruiz, K. S. Fernando, B. Wang, N. A. Brown, P. G. Luo, N. D. McNamara, M. Vangsness, Y.-P. Sun and C. E. Bunker, ACS Nano, 2011, 5, 8100-8107.

4 E. C. Qin, M. E. Kandel, E. Liamas, T. B. Shah, C. Kim, C. D. Kaufman, Z. J. Zhang, G. Popescu, M. U. Gillette and D. E. Leckband, Acta Biomater., 2019, 90, 412-423.

5 M. Lv, Y. Zhang, L. Liang, M. Wei, W. Hu, X. Li and Q. Huang, Nanoscale, 2012, 4, 3861-3866.

6 P. Li, T. Xu, S. Wu, L. Lei and D. He, J. Appl. Toxicol., 2017, 37, 1140-1150.

7 X. Hu, Z. Wei and L. Mu, Carbon, 2017, 117, 182-191.

8 Y. Li, H. Yuan, A. Von Dem Bussche, M. Creighton, R. H. Hurt, A. B. Kane and H. Gao, Proc. Natl. Acad. Sci. U. S. A., 2013, 110, 12295-12300.

9 W. Zhang, C. Wang, Z. Li, Z. Lu, Y. Li, J. J. Yin, Y. T. Zhou, X. Gao, Y. Fang and G. Nie, Adv. Mater., 2012, 24, 53915397.

10 M. Zhou, X. Gong, Y. Wang, C. Liu, M. Hong, L. Wang and F. Hong, Biol. Trace Elem. Res., 2011, 142, 760-772.

11 X. Zou, L. Zhang, Z. Wang and Y. Luo, J. Am. Chem. Soc., 2016, 138, 2064-2077.

12 I. E. M. Carpio, C. M. Santos, X. Wei and D. F. Rodrigues, Nanoscale, 2012, 4, 4746-4756.

13 H. Zhu, L. Gao, X. Jiang, R. Liu, Y. Wei, Y. Wang, Y. Zhao, Z. Chai and X. Gao, Chem. Commun., 2014, 50, 36953698.

14 T. Palaniselvam, H. B. Aiyappa and S. Kurungot, J. Mater. Chem., 2012, 22, 23799-23805.

15 Q. Tu, L. Pang, Y. Chen, Y. Zhang, R. Zhang, B. Lu and J. Wang, Analyst, 2014, 139, 105-115.

16 A. Sasidharan, L. Panchakarla, P. Chandran, D. Menon, S. Nair, C. Rao and M. Koyakutty, Nanoscale, 2011, 3, 24612464.

17 H. Pieper, S. Chercheja, S. Eigler, C. E. Halbig, M. R. Filipovic and A. Mokhir, Angew. Chem., Int. Ed., 2016, 55, 405-407.

18 Q. Zhang, X. Liu, H. Meng, S. Liu and C. Zhang, Environ. Sci.: Nano, 2018, 5, 1361-1371.

19 Y. Tu, M. Lv, P. Xiu, T. Huynh, M. Zhang, M. Castelli, Z. Liu, Q. Huang, C. Fan and H. Fang, Nat. Nanotechnol., 2013, 8, 594.

20 N. Smirnoff, Vitam. Horm., 2001, 61, 241-266.

21 M. Sinha, P. Manna and P. C. Sil, BMB Rep., 2008, 41, 657663.

22 Y. Li, Y. Liu, Y. Fu, T. Wei, L. Le Guyader, G. Gao, R.-S. Liu, Y.-Z. Chang and C. Chen, Biomaterials, 2012, 33, 402-411.

23 A. Sasidharan, S. Swaroop, P. Chandran, S. Nair and M. Koyakutty, Nanomedicine, 2016, 12, 1347-1355.

24 S. Zhou, Y. Wang and J.-J. Zhu, ACS Appl. Mater. Interfaces, 2016, 8, 7674-7682.

25 X. Lu, X. Feng, J. R. Werber, C. Chu, I. Zucker, J.-H. Kim, C. O. Osuji and M. Elimelech, Proc. Natl. Acad. Sci. U. S. A., 2017, 114, E9793-E9801. 